\title{
Lung cancer and breast cancer mortality trends among 45-74-year-old European women
}

\author{
Urszula Sulkowska1, Irmina Maria Michałek', Joanna Didkowska1,2, Paweł Koczkodaj²
}

\author{
'National Cancer Registry, Maria Sklodowska-Curie National Research Institute of Oncology, Warsaw, Poland \\ ${ }^{2}$ Cancer Epidemiology and Primary Prevention Department, Maria Sklodowska-Curie National Research Institute of Oncology, Warsaw, Poland
}

\begin{abstract}
Introduction. We aimed to analyze and compare the most up-to-date breast and lung cancer mortality rates in European women aged 45-74.

Material and methods. The data on breast and lung cancer mortality in 1960-2017 were obtained from the World Health Organization Mortality Data Base and Eurostat. To determine the mortality trends and generate annual percent change, with $95 \%$ confidence intervals, joinpoint regression was applied.

Results. In most European Union (EU) member states (15 out of 28), lung cancer mortality was higher than breast cancer mortality, with either increasing or stable lung cancer mortality rates. In four other EU countries, breast and lung cancer mortality rates in the last reported year were almost equal or equal.

Conclusions. Lung cancer is becoming the leading cause of cancer deaths among European women. There is a need for ensuring women-targeted smoking cessation services to decrease tobacco-attributable lung cancer mortality.
\end{abstract}

Key words: lung cancer, breast cancer, women, mortality, tobacco, cancer prevention, Europe

\section{Introduction}

Breast cancer is the most prevalent female neoplasm worldwide. According to the International Agency for Research on Cancer (IARC), in 2018, globally, 2,261,419 women were diagnosed with breast cancer. Moreover, breast cancer is a leading cause of cancer deaths among women (684,996 deceases in 2018). According to the same global cancer statistics, lung cancer is the third-most-common female neoplasm and the second-most-common cause of female cancer deaths globally, with the number of incident cases at 770,828 and the number of deaths at 607,465 in 2018 [1]. In the European Union (EU; state of 2018 with $28 \mathrm{EU}$ member states), breast cancer is still the most prevalent female neoplasm, however, lung cancer is now the leading cause of female cancer deaths [2].
While a systematic understanding of breast cancer risk factors is still unsatisfactory, it is already known that about 70-80\% of female lung cancer cases are associated exclusively to tobacco smoking $[3,4]$. Hence, cancer mortality trends are affected by changes in European tobacco consumption patterns. At the end of the $20^{\text {th }}$ century, tobacco-related mortality decreased among men, and was stable or increased among women [5]. This phenomenon is evident in lung cancer, considered a good proxy for smoking prevalence estimations.

Although mechanisms underpinning cancer prevalence and mortality rates are not fully understood, their changes can be a valuable indicator for policymakers and stakeholders, enabling more tailored and efficient actions aimed at

\section{How to cite:}

Sulkowska U, Michałek IM, Didkowska J, Koczkodaj P. Lung cancer and breast cancer mortality trends among 45-74-year-old European women. NOWOTWORY J Oncol 2021; 71: 185-196.

This article is available in open access under Creative Common Attribution-Non-Commercial-No Derivatives 4.0 International (CC BY-NC-ND 4.0) license, allowing to download articles and share them with others as long as they credit the authors and the publisher, but without permission to change them in any way or use them commercially. 
decreasing tobacco consumption in the EU and its suitable member states. This study aimed to analyze and compare the most recent female breast and lung cancer mortality rates in 31 European countries.

\section{Material and methods}

The presented analysis is an update of the data published in the article by Sulkowska et al. in Nowotwory. Journal of Oncology 2015; 65 (5): 395-403, entitled Lung cancer, the leading cause of cancer deaths among women in Europe [5]. We followed previously applied methodology (including the same age group: 45-74 years old) to enable comparability of the data.

\section{Source of the data}

The analyzed data were obtained in a hybrid manner. First, we obtained data from the World Health Organization (WHO) Mortality Data Base (MDB) (data available as of $15^{\text {th }}$ December 2019). The MDB contains the number of deaths by country, year, sex, age group, and cause of death. The cause of death is coded according to the International Classification of Diseases (ICD). We identified all female deaths due to breast and lung cancer registered in the MDB since 1960 in 28 EU member states and three non-member states, namely Norway, Russia, and Switzerland. The included diagnosis codes encompassed lung cancer (162-163 - ICD $7^{\text {th }}$ revision; $162-$ ICD $8^{\text {th }}$ and $9^{\text {th }}$ revisions; and C33 and C34 - ICD $10^{\text {th }}$ revision) and breast cancer $\left(170-7^{\text {th }}\right.$ revision; $174-8^{\text {th }}$ and $9^{\text {th }}$ revisions; and C50 $10^{\text {th }}$ revision). In cases where the data for additional (following) years were available in Eurostat, these were also included in our analysis (detailed data sources, by country, by year in table I). The mid-year population estimates were obtained from WHO MDB and Eurostat.

\section{Statistical analysis}

Crude annual mortality rates were defined as the number of new deaths per 100,000 person-years. In the denominator, we applied the mid-year population, defined as the population's size on the $31^{\text {st }}$ of June. In all calculations, both the numerator and denominator came from the same data source, WHO MDB or Eurostat. To enable a comparison with other populations, we performed direct age-standardization for the Segi's World Standard Population [6]. For Luxembourg and Malta, the mortality rates were calculated as three-year moving averages (deploying the preceding and following year).

To determine mortality trends and to generate the annual percent change (APC), with 95\% confidence intervals (CI), joinpoint regression was applied [7]. The best-fitting model was selected with permutations tests, with an overall significance level at 0.05 and the number of randomly permutated data sets for permutation set at 4499. Rates were considered to decrease if $\mathrm{APC}<0$ and $95 \% \mathrm{Cl}$ does not contain zero, and to increase if $\mathrm{APC}>0$ and $95 \% \mathrm{Cl}$ do not contain zero; otherwise, rates were considered stable.
Joinpoint analysis was performed using the Joinpoint Regression Program (version 4.3.1.0, National Cancer Institute, Bethesda, MD, USA).

\section{Compliance with ethical standards}

According to the $\mathrm{WHO}$ and Eurostat policies, the analyzed data can be freely used for scientific purposes. This study was conducted according to the Strengthening the Reporting of Observational Studies in Epidemiology (STROBE) guidelines [8].

\section{Results}

Breast and lung cancer mortality rates in 1960-2017, analyzed by EU member states, manifested four different patterns:

- Group 1 - higher mortality from lung cancer than from breast cancer with increasing mortality rates of lung cancer;

- Group 2 - higher mortality from lung cancer than from breast cancer with stable or decreasing lung cancer mortality rates;

- Group 3 - almost equal or equal breast and lung cancer mortality rates in the last reported year;

- Group 4 - other EU countries (tab. I).

Non-EU countries were analyzed separately, as Group 5. In the vast majority of countries in group 1, lung and breast cancer mortality rates intersected around 2010. In Poland the intersection occurred in 2004, and in Spain in 2016 (fig. 1 A). In Austria, Croatia, Germany, and Slovenia, the increase in lung cancer mortality rates was constant. In Poland, a very short period of trend stabilization was observed between 1968-1972, and in Luxembourg, lung cancer mortality rates decreased between 1971-1974. In Czechia, the trend began stabilizing in 2000. In Spain, in 1990, after years of a plateau, lung cancer mortality rates started increasing.

In group 2, time of the lung and breast mortality trends intersection varied widely, e.g., in Denmark it took place in 1991, in Sweden in 2001, and in Ireland in 2012 (fig. 1 B). Lung cancer mortality rates were sharply dropping in Belgium, Denmark, Sweden, and the United Kingdom. In Hungary, Ireland, and the Netherlands, the decrease was more gradual. The onset of decreasing rates for lung cancer mortality ranged from 1980 in Ireland to 2015 in Belgium.

In group 3, the breast and lung cancer mortality rates were almost equal or equal (fig. 1 C). In all countries in the group, lung cancer mortality increased; however, only in Italy was the increase constant. The trend plateaued in Finland and France in 1962-1974 and in 1960-1977, respectively.

In every country in group 4, lung cancer mortality has always been lower than breast cancer mortality (fig. 1 D). However, in some countries (Bulgaria, Cyprus, Estonia, Lithuania, Malta, Portugal, and Romania), the breast cancer mortality rate has been decreasing substantially and/or the lung cancer mortality rate has been sharply increasing, which might point toward future intersection of the rates. 


\begin{tabular}{|c|c|c|c|}
\hline$\frac{7}{3}$ & $\frac{7}{3}$ & 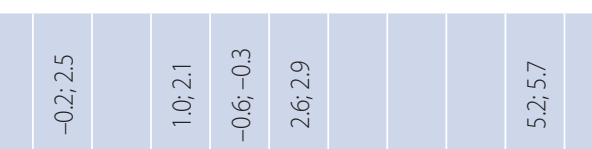 & 总 \\
\hline के & $\stackrel{\square}{=}$ & 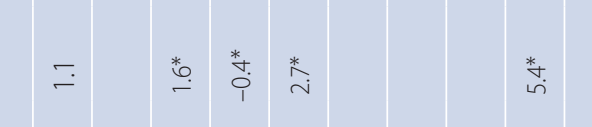 & 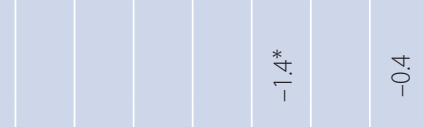 \\
\hline$\frac{\frac{2}{2}}{\frac{2}{2}}$ & & 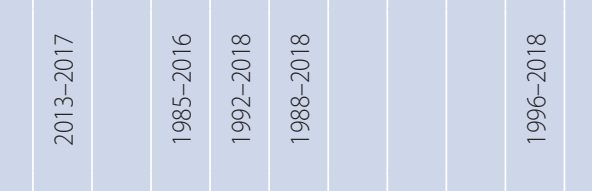 & \\
\hline$\frac{7}{3}$ & & 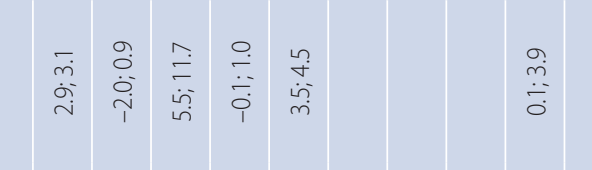 & 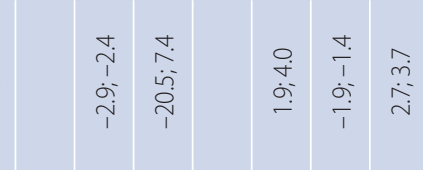 \\
\hline$\$$ & & 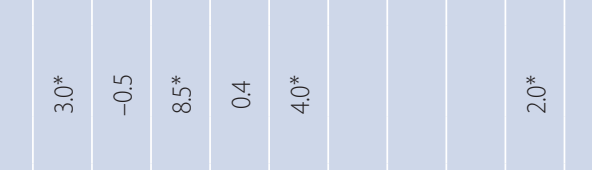 & $3 \div$ \\
\hline 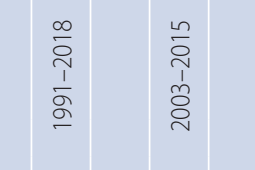 & & 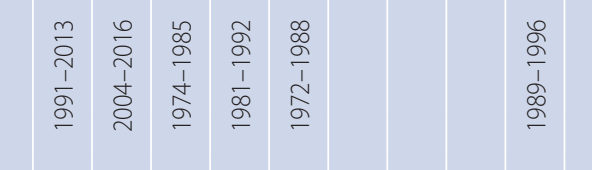 & 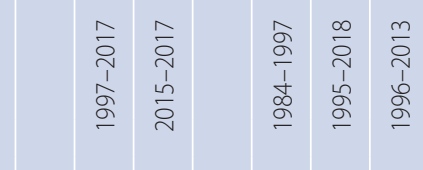 \\
\hline 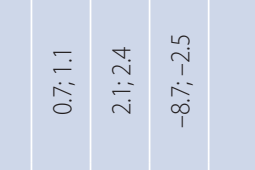 & $\frac{2}{3}$ & 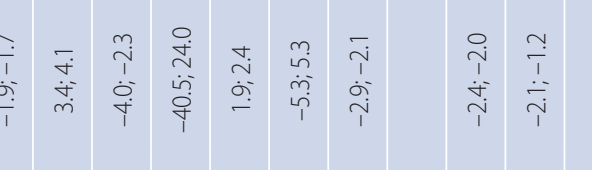 & 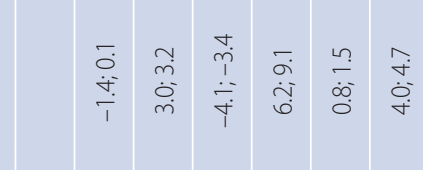 \\
\hline $5: 5$ & $\bar{s}:$ & 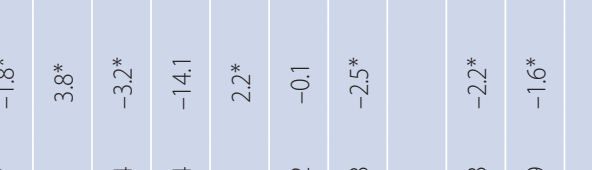 & 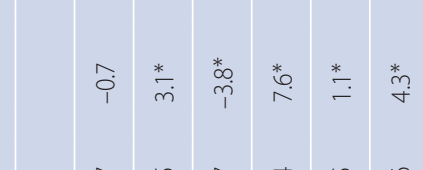 \\
\hline 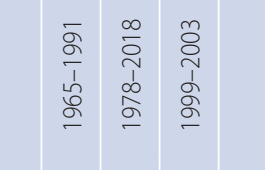 & 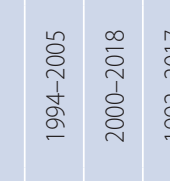 & 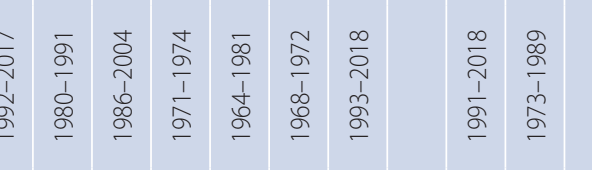 & 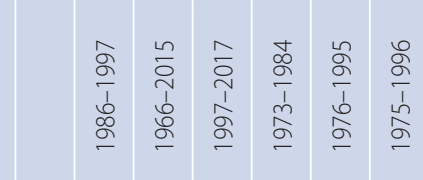 \\
\hline 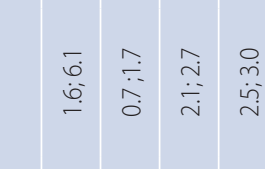 & 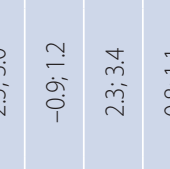 & 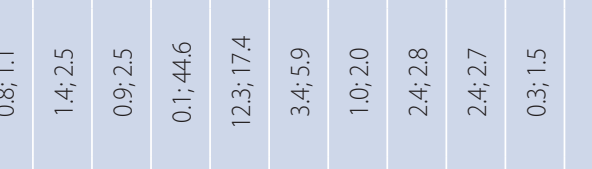 & 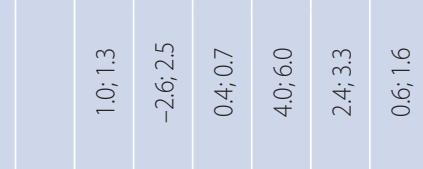 \\
\hline$\dot{m}=3$ & $\bar{\alpha}$ & 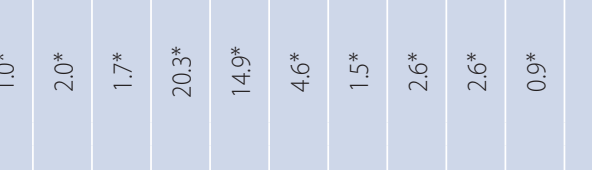 & $\fallingdotseq \bar{g} \dot{\bar{g}}$ \\
\hline 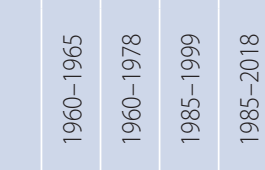 & & 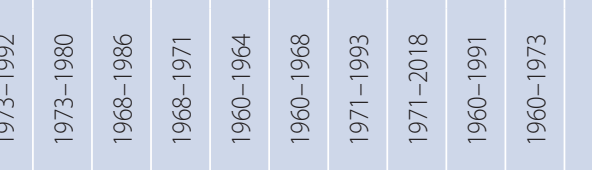 & 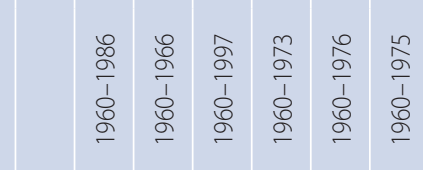 \\
\hline 天气 & & 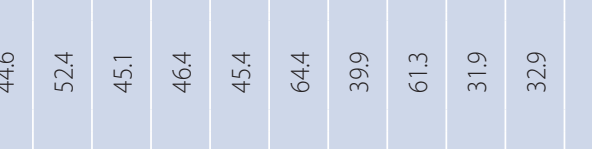 & 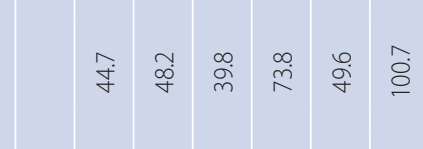 \\
\hline 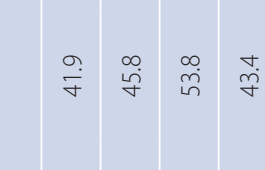 & & 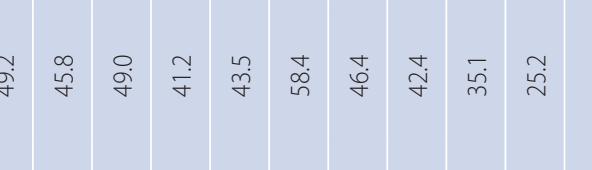 & 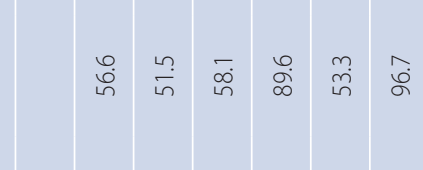 \\
\hline 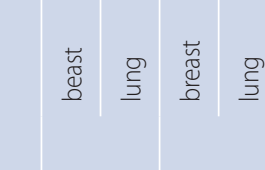 & & 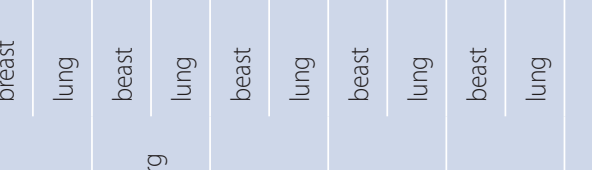 & \\
\hline
\end{tabular}




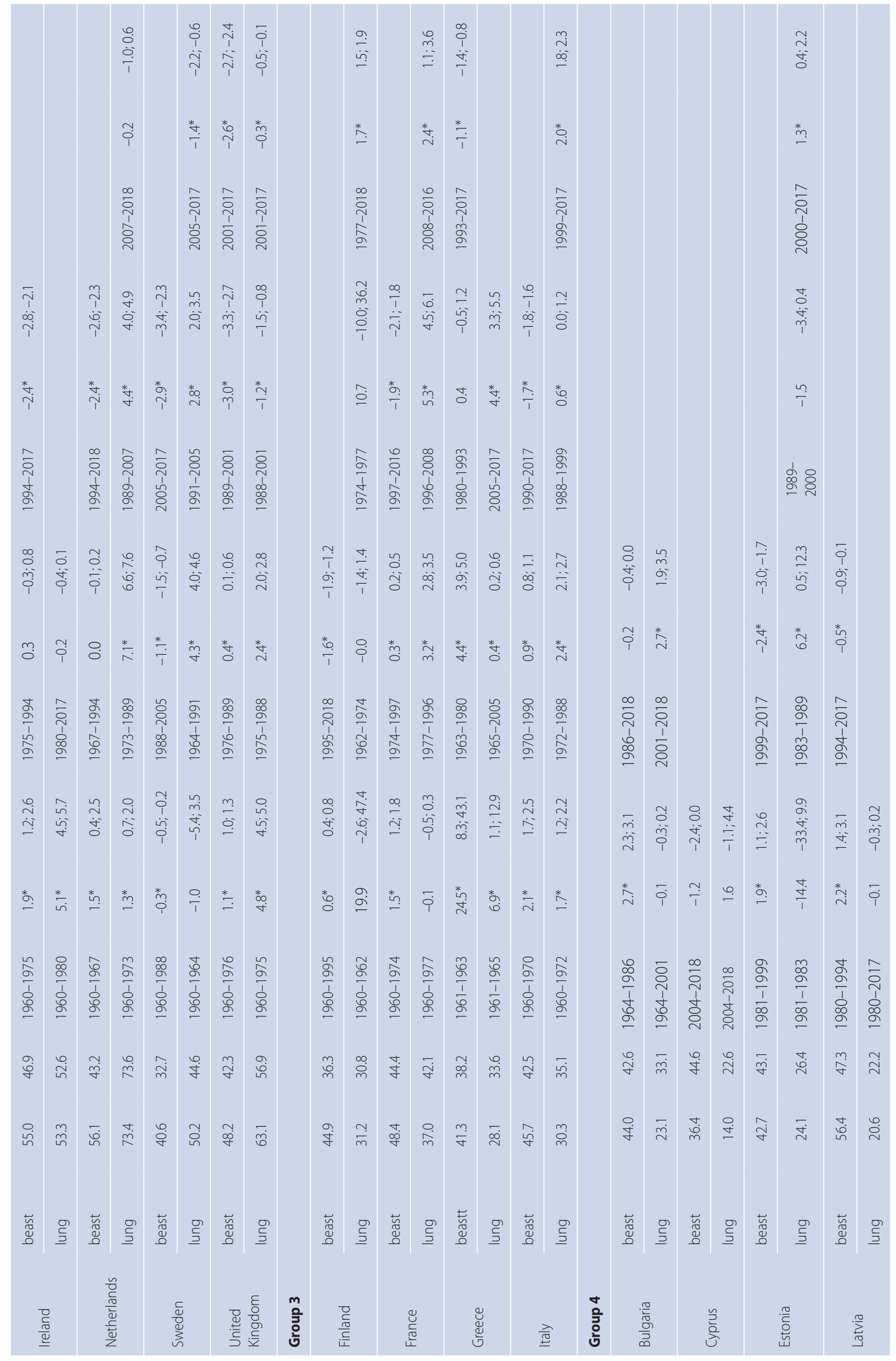




\begin{tabular}{|c|c|c|c|c|c|c|c|c|c|c|}
\hline & 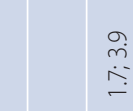 & 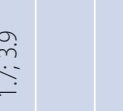 & & & & & & & & 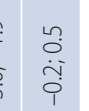 \\
\hline & $\stackrel{\infty}{\sim}$ & . & & & & & & & & $\overline{0}$ \\
\hline & 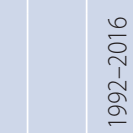 & 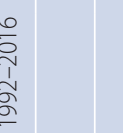 & & & & & & & & 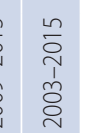 \\
\hline 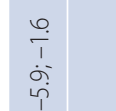 & 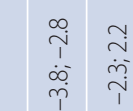 & 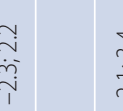 & 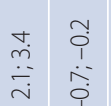 & & 吕 & & $\begin{array}{l}\infty \\
\stackrel{0}{0} \\
\stackrel{0}{0}\end{array}$ & & & $\begin{array}{l}n \\
\substack{n \\
\infty}\end{array}$ \\
\hline 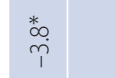 & ${ }_{\bar{p}}^{*} \bar{i}$ & $\bar{i}$ & 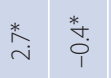 & & sa & & ฮี & & & 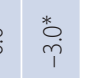 \\
\hline 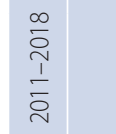 & 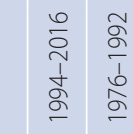 & 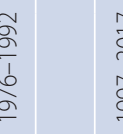 & 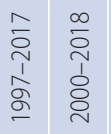 & & 常 & & 彥 & & & 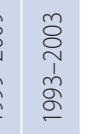 \\
\hline 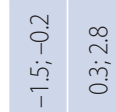 & \begin{tabular}{l}
0 \\
\hdashline \\
\hdashline \\
\hdashline
\end{tabular} & 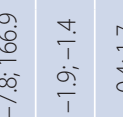 & & 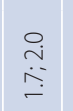 & $\begin{array}{ll}\bar{c} \\
0 \\
0\end{array}$ & & 品 & 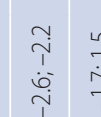 & & $\frac{\sim}{\bar{\tau}}$ \\
\hline 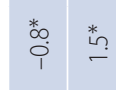 & $=\overline{0}$ & 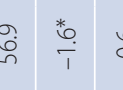 & $\stackrel{*}{\circ} \stackrel{*}{=}$ & $\stackrel{\$}{\infty}$ & 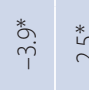 & & $\vdots$ & 索 & & $\hat{i}$ \\
\hline 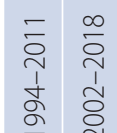 & 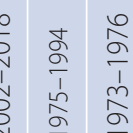 & 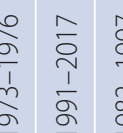 & 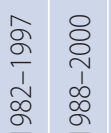 & $\begin{array}{c}\infty \\
\vdots \\
\vdots \\
\vdots \\
\vdots\end{array}$ & 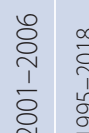 & & $\frac{\bar{g}}{\frac{\delta}{a}}$ & 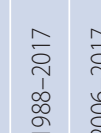 & & 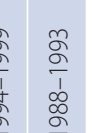 \\
\hline 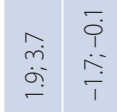 & {$\left[\begin{array}{c}\infty \\
\infty \\
\infty \\
\vdots\end{array}\right.$} & $\frac{m}{m}=\frac{m}{m}$ & & $\overline{\dot{u}}$ & 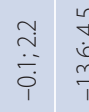 & & & 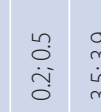 & & 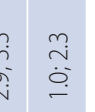 \\
\hline$\stackrel{\text { so }}{\sim} \underset{\sim}{*} \stackrel{*}{Q}$ & 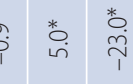 & to & ث & \$ & $\stackrel{5}{-}$ & & $\approx$ & 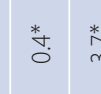 & & $\stackrel{t}{0}$ \\
\hline 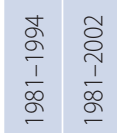 & 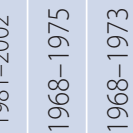 & 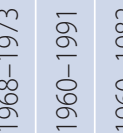 & 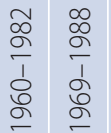 & 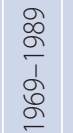 & 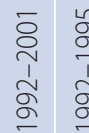 & & $\frac{2}{8}$ & 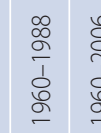 & & \\
\hline 字 & $=\bar{\sigma} \bar{\sigma}$ & $\left.\mathbb{r}^{\infty}\right)^{\infty}$ & $\stackrel{\infty}{\sim} \underset{\sim}{\stackrel{\infty}{f}}$ & $\bar{m}$ & $\frac{\circ}{\sigma}$ & & 声 & $\stackrel{\circ}{\circ}$ & & $\stackrel{\infty}{=}$ \\
\hline$\overline{\bar{n}} \stackrel{n}{n}$ & $=8$ & స̃ & $\stackrel{m}{=} \frac{a}{q}$ & $\stackrel{\circ}{\sim}$ & $\begin{array}{l}\% \\
q\end{array}$ & & 总 & 急 & & $\stackrel{m}{2}$ \\
\hline 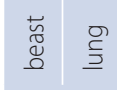 & 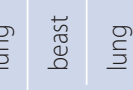 & $\frac{0}{5}$ & 兽 & 豆 & $\begin{array}{l}\mathrm{s} \\
\mathrm{g}\end{array}$ & & 号 & 憘 & & 党哭 \\
\hline 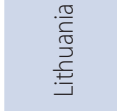 & $\frac{\frac{\pi}{5}}{2}$ & 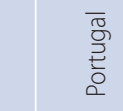 & & & 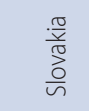 & 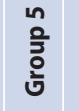 & & 离 & & 离 \\
\hline
\end{tabular}


A

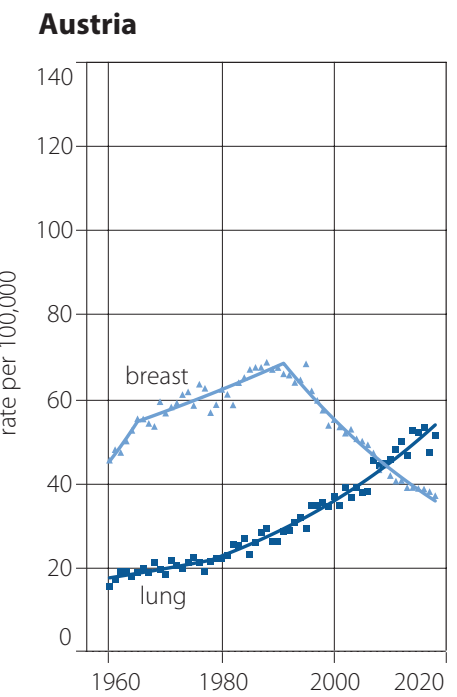

Germany

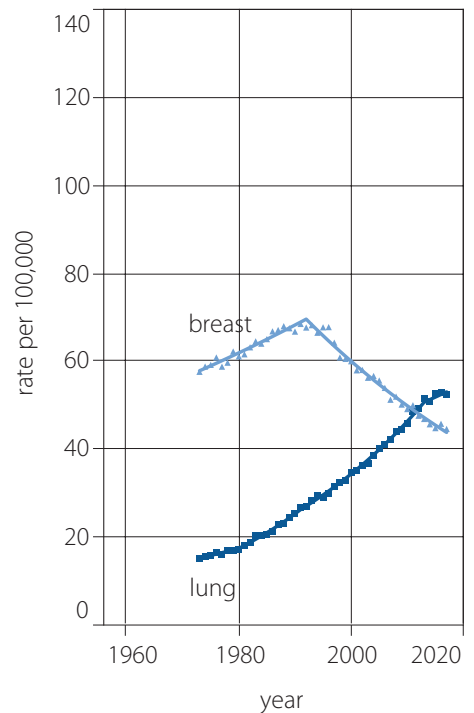

Slovenia

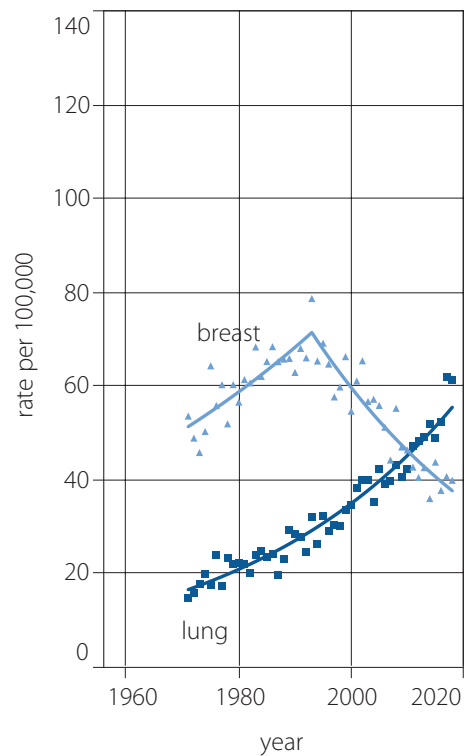

\section{Croatia}

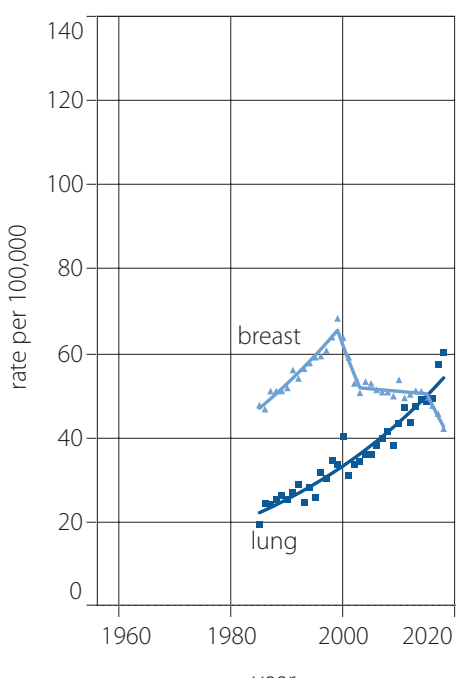

Luxembourg

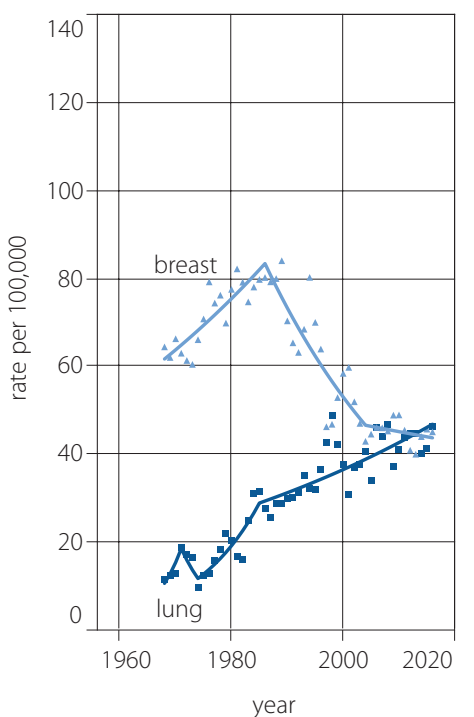

\section{Spain}

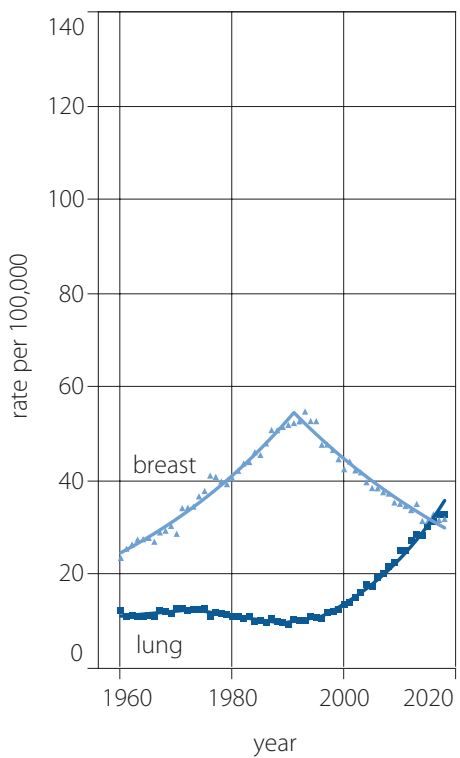

\section{Czechia}

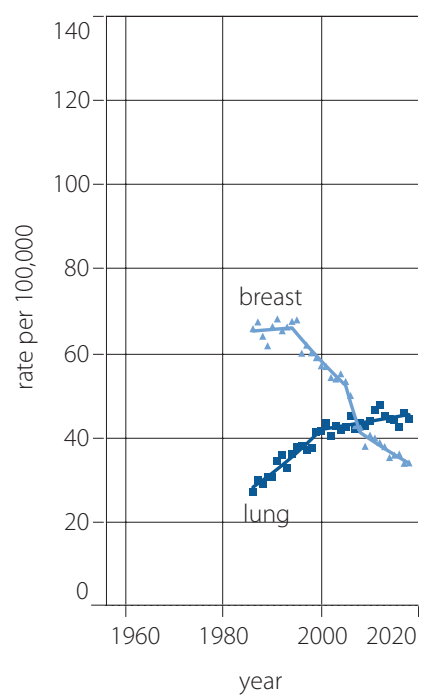

Poland

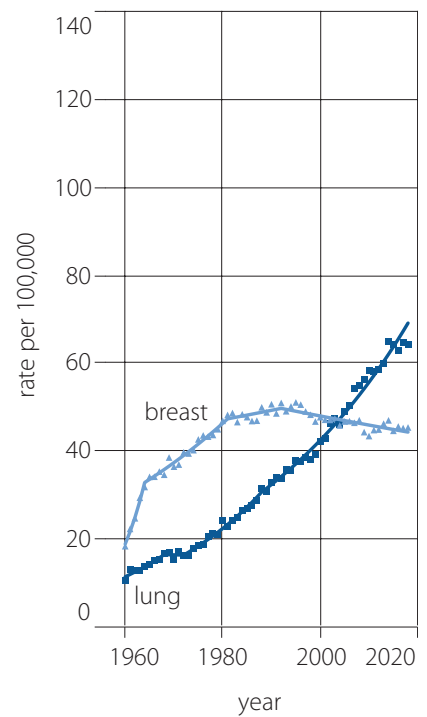

Figure 1. A. Breast and lung cancer mortality rates among women aged 45-74-years-old. Group 1 - EU countries with higher mortality from lung cancer than from breast cancer with increasing lung cancer mortality rates 


\section{Belgium}

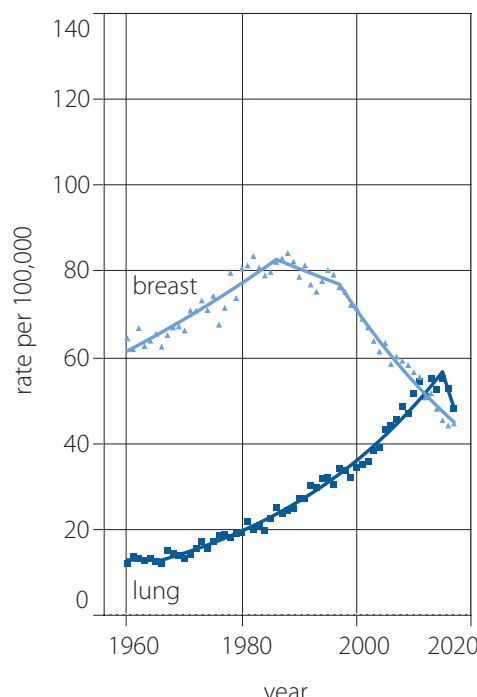

\section{Ireland}

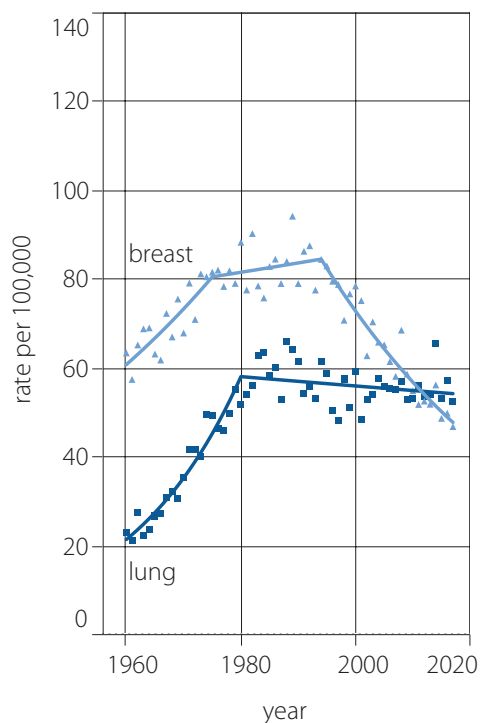

United Kingdom

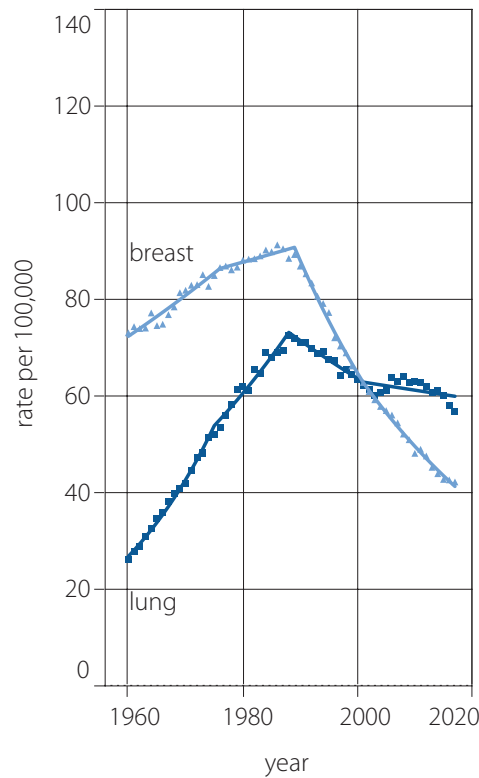

\section{Denmark}
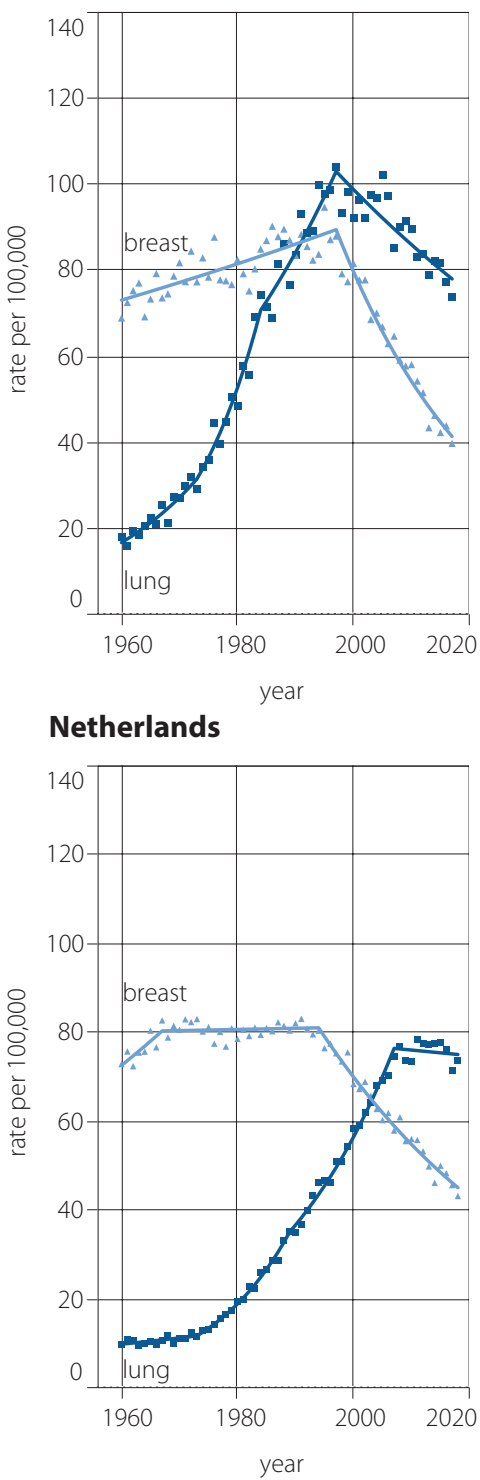

\section{Hungary}

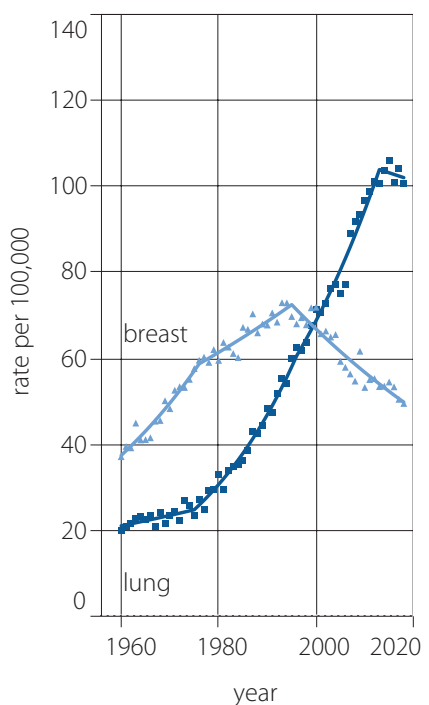

\section{Sweden}

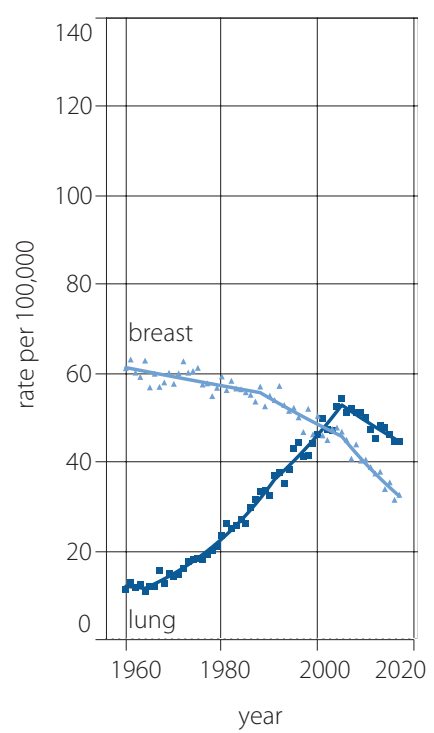


C

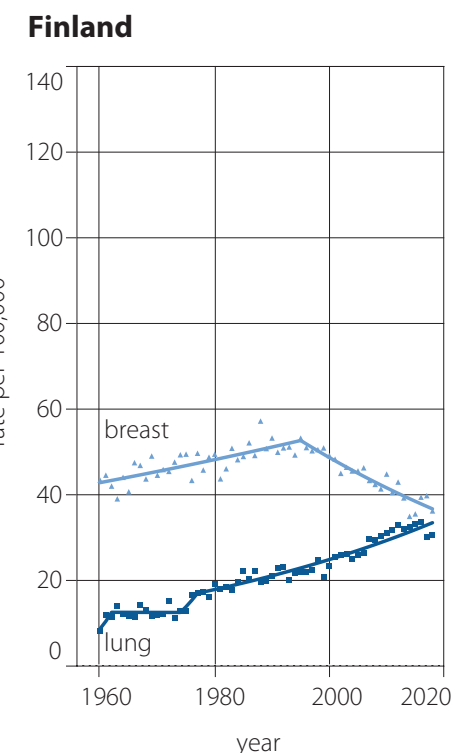

\section{Italy}

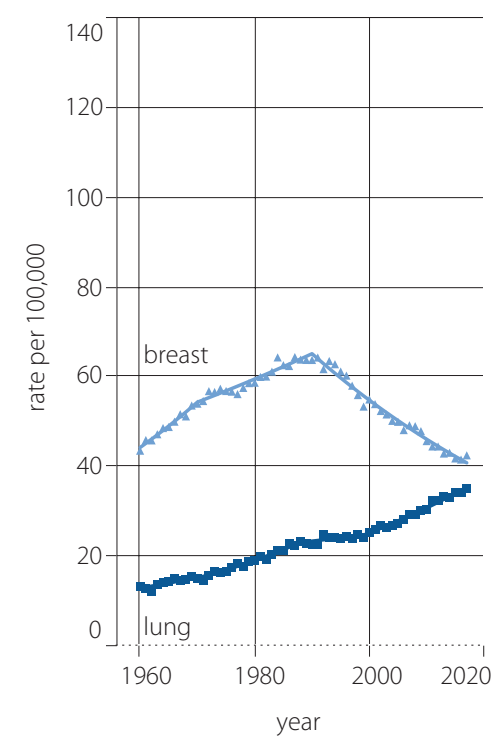

\section{France}

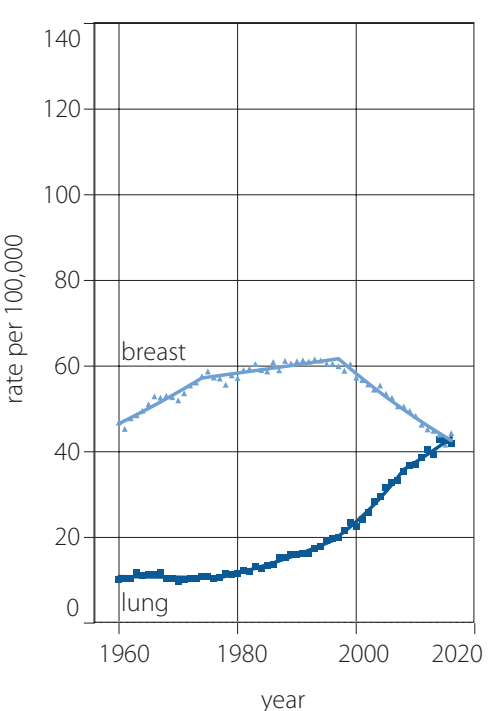

\section{Greece}

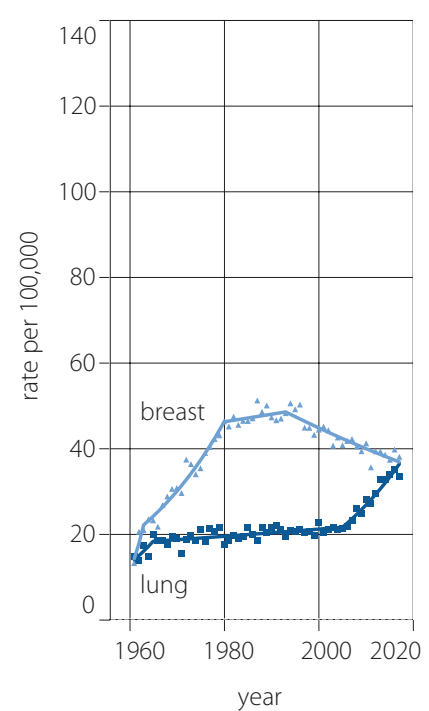

Figure 1. C. Breast and lung cancer mortality rates among women aged 45-74-years-old. Group 3 - EU countries with almost equal or equal breast and lung cancer mortality rates in the last reported year 
D

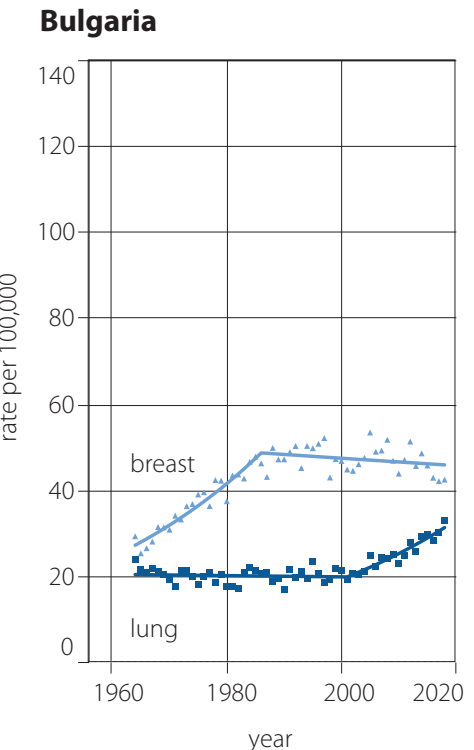

Latvia

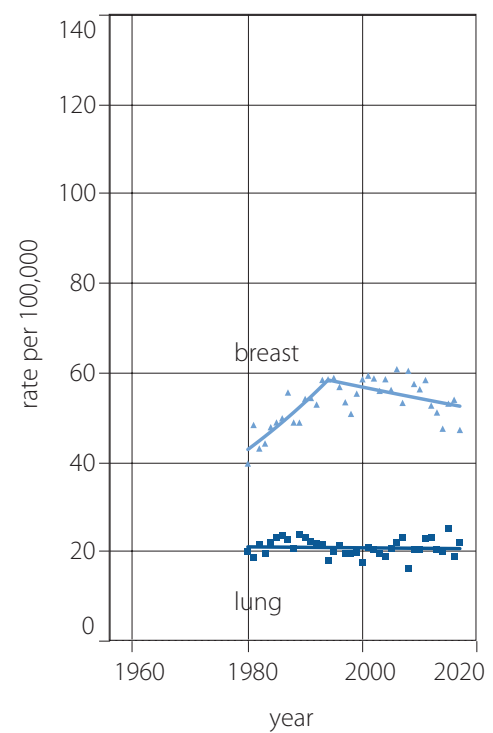

Portugal

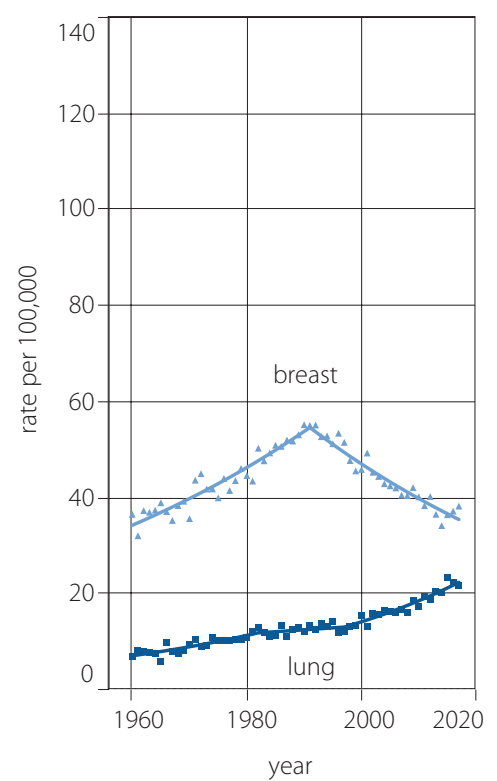

\section{Cyprus}

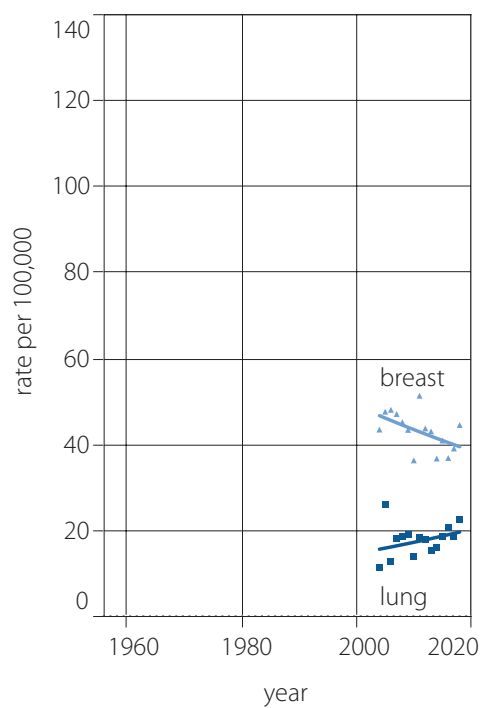

Lithuania

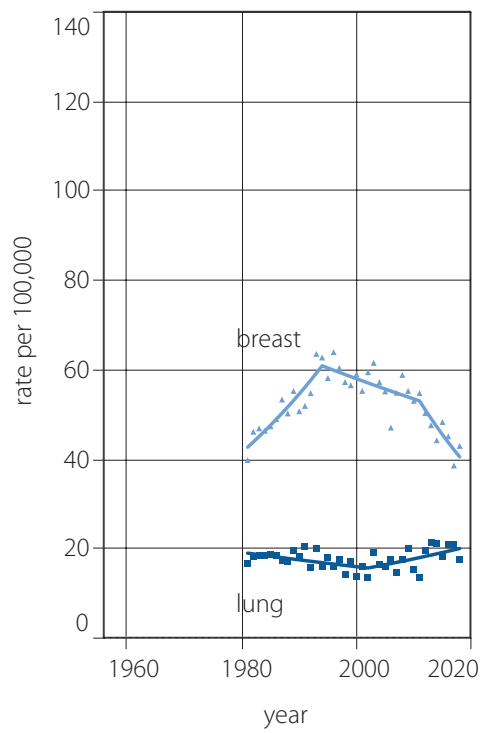

Romania

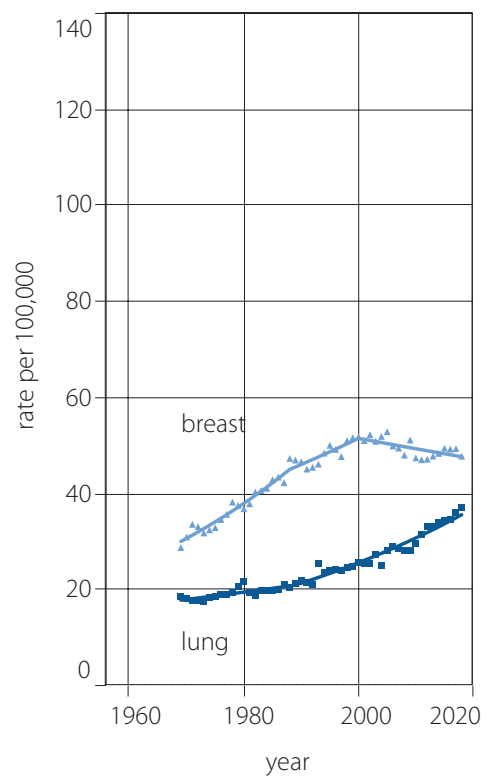

\section{Estonia}

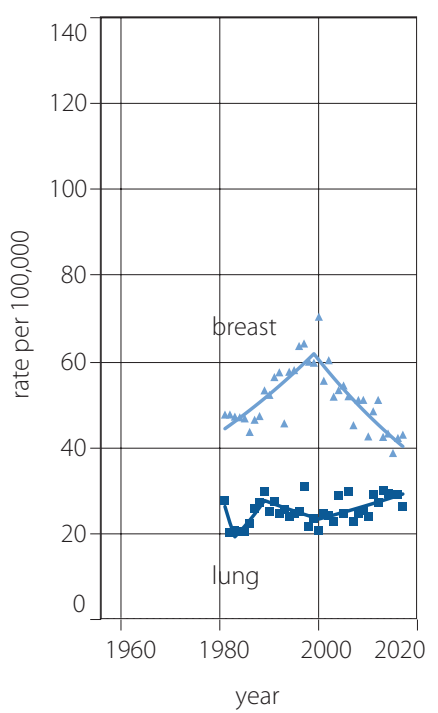

Malta

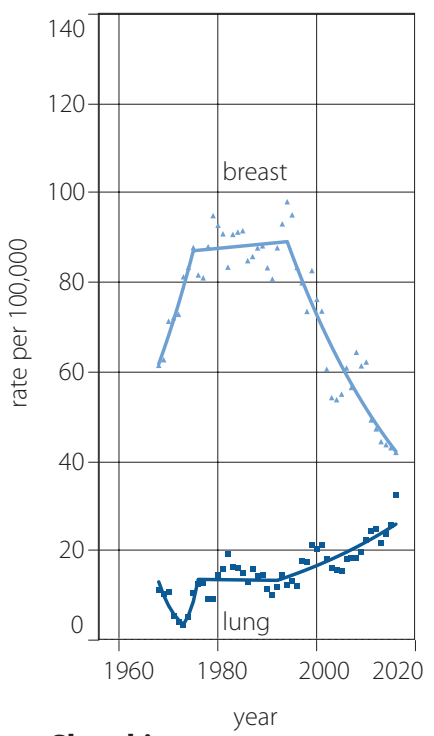

Slovakia

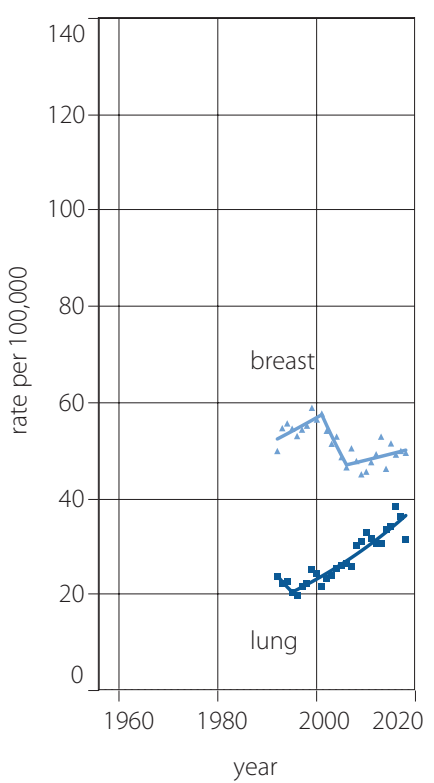

Figure 1. D. Breast and lung cancer mortality rates among women aged 45-74-years-old. Group 4 - other EU countries 
E

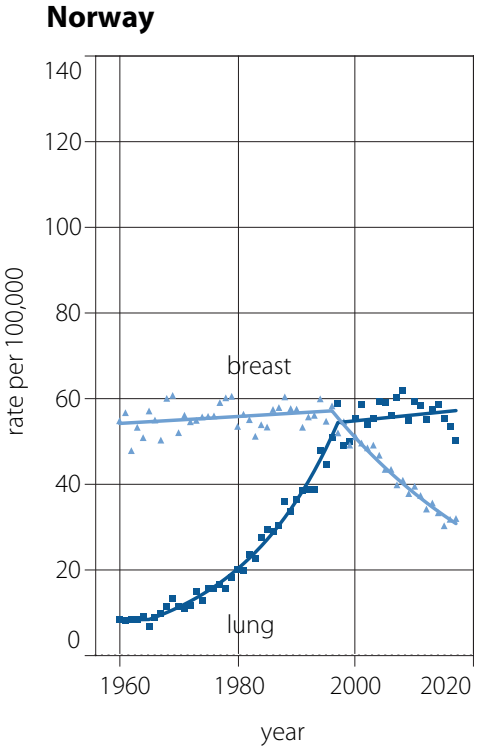

\section{Russia}

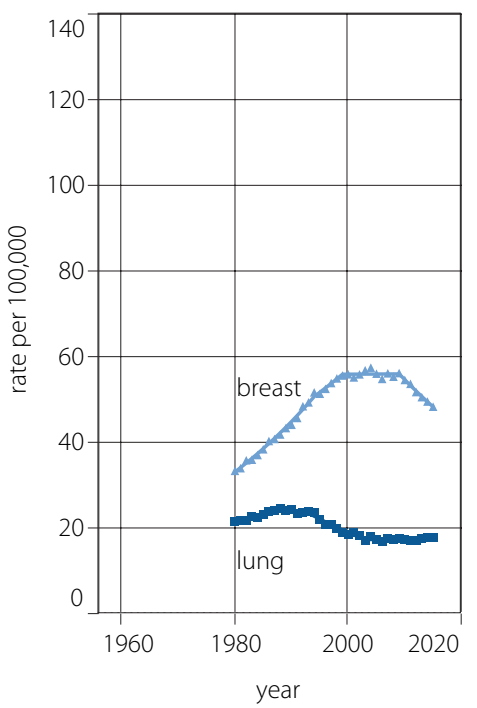

Switzerland

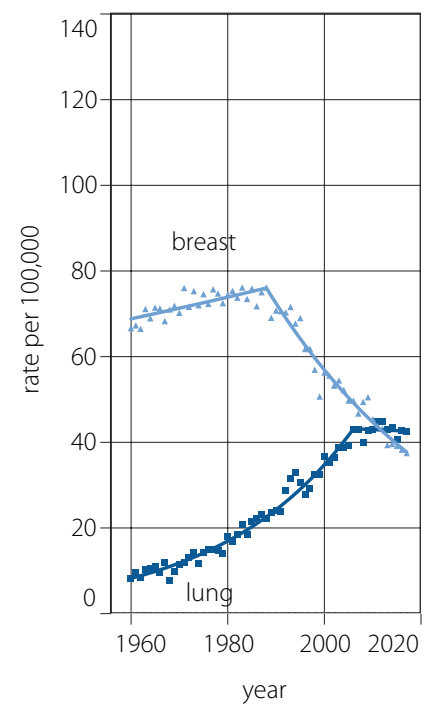

Figure 1. E. Breast and lung cancer mortality rates among women aged 45-74-years-old. Group 5 - non-EU countries

Group 5 represents three non-EU countries (fig. 1 E). In Norway and Switzerland the rates intersected in 1998 and 2012, respectively. In Russia, such a phenomenon has never occurred.

\section{Discussion}

The presented analysis depicts a substantial increase in female lung cancer mortality across the vast majority of European countries (tab. II). In comparison with our previous analysis on female lung and breast cancer mortality in the EU [5] (the last reported year was 2010), we noticed progressive cancer mortality changes. Previously we had forecasted further increases in lung cancer mortality and the intersection of both analyzed trends for 12 EU countries. This forecast proved to be true for Belgium, Croatia, Spain, Ireland, Germany, and Slovenia, in our current analysis. However, in Finland, France, Greece, and Italy, the trends have not intersected yet. Contrary to our earlier predictions, the current analysis shows that in Estonia and Slovakia breast cancer mortality is still higher than lung cancer mortality.

Considering the most up-to-date data on tobacco use, we know that at present in the EU about 47 million women currently smoke. Moreover, the advanced stage of tobacco epidemic was observed in 12 UE member states, where smoking prevalence among women is higher than 15\% [9]. According to the Institute for Health Metrics and Evaluation (IHME), an exceptionally high smoking-attributable disease burden is observed in Bulgaria, Croatia, Greece, Hungary, and Poland, with the disability-adjusted life years index ranging between 17.5\% and 20\% [10]. Trends reported in our analysis are following the IHME data. Noteworthy, in Poland and Croatia, the increase is very sharp, and Hungary is characterized by the highest lung cancer mortality rate among all 31 analyzed countries (>100 per 100,000).

The presented analysis implies that greater efforts are needed to ensure a decline in lung cancer mortality rates. Several possible courses of action are mainly related to more restrictive anti-tobacco policies. Raising the excise tax for tobacco products is one of the most effective tools to achieve this goal [11], particularly among women who are more responsive to such measures than men [12]. Another solution is banning menthol and slim cigarettes, perceived as being more feminine tobacco products, targeted primarily at this group of users $[13,14]$. Some of these solutions have already been introduced under the Tobacco Products Directive (2014/40/ EU) [15]. However, the decline in lung cancer mortality observed in our analysis should not yet be connected with the enforcement of this particular law, since it has been in force too short to impact the mortality statistics. Notwithstanding, effective implementation of the Directive should be a priority for European policymakers, since it may further reduce lung cancer mortality among EU women.

The strength of the analysis is in the completeness of the analyzed cause-of-death data, which was close to 100\%, except for Cyprus, where it was 68\% [16]. The most important limitation of the study results from the possible cross-national differences in coding practices, particularly in codes for ill-defined and unknown causes. This should be taken into account when comparing mortality rates for specific causes 
Table II. Completeness $\uparrow$ of cause-of-death data and their source by years included

\begin{tabular}{|c|c|c|c|}
\hline \multirow[t]{2}{*}{ Country } & \multicolumn{2}{|c|}{ WHO MDB } & \multirow{2}{*}{$\begin{array}{c}\text { Eurostat } \\
\begin{array}{c}\text { Years } \\
\text { included }\end{array}\end{array}$} \\
\hline & Years included & Completeness & \\
\hline Austria & 1960-2017 & $100 \%$ & 2018 \\
\hline Belgium & 1960-2016 & $100 \%$ & 2017 \\
\hline Bulgaria & 1964-2015 & $100 \%$ & $2016-2018$ \\
\hline Croatia & 1985-2016 & $100 \%$ & $2017-2018$ \\
\hline Cyprus & 2004-2016 & $68 \%$ & 2017-2018 \\
\hline Czechia & 1986-2017 & $100 \%$ & 2018 \\
\hline Denmark & 1960-2015 & $100 \%$ & 2016-2017 \\
\hline Estonia & 1981-2016 & $100 \%$ & 2017 \\
\hline Finland & 1960-2016 & $100 \%$ & $2017-2018$ \\
\hline France & 1960-2015 & $100 \%$ & 2016 \\
\hline Germany & 1973-2016 & $100 \%$ & 2017 \\
\hline Greece & $1961-2016$ & $100 \%$ & 2017 \\
\hline Hungary & 1960-2017 & $100 \%$ & 2018 \\
\hline Ireland & 1960-2015 & $100 \%$ & 2016-2017 \\
\hline Italy & $1960-2015$ & $100 \%$ & 2016-2017 \\
\hline Latvia & 1980-2015 & $100 \%$ & 2016-2017 \\
\hline Lithuania & 1981-2017 & $99 \%$ & 2018 \\
\hline Luxembourg & 1968-2016 & $100 \%$ & 2017 \\
\hline Malta & 1968-2015 & $100 \%$ & 2016-2017 \\
\hline Netherlands & 1960-2016 & $100 \%$ & 2017-2018 \\
\hline Norway & 1960-2016 & $100 \%$ & 2017 \\
\hline Poland & 1960-2016 & $100 \%$ & $2017-2018$ \\
\hline Portugal & 1960-2016 & $100 \%$ & 2017 \\
\hline Romania & 1969-2017 & $100 \%$ & 2018 \\
\hline Russia & 1980-2015 & $100 \%$ & - \\
\hline Slovakia & 1992-2014 & $100 \%$ & 2015-2018 \\
\hline Slovenia & 1971-2015 & $100 \%$ & 2016-2018 \\
\hline Spain & $1960-2016$ & $100 \%$ & $2017-2018$ \\
\hline Sweden & 1960-2016 & $100 \%$ & 2017 \\
\hline Switzerland & 1960-2016 & $100 \%$ & 2017 \\
\hline United Kingdom & 1960-2016 & $100 \%$ & 2017 \\
\hline
\end{tabular}

t- available for the WHO MDB only; WHO MDB - the World Health Organization Mortality Data Base

across countries. However, since we assessed time trends of mortality rates within the countries in this study, the presented results' generalizability should not be limited.

\section{Conclusions}

In many European countries during the last decades, lung cancer has become the leading cause of cancer deaths among women. Ensuring the implementation of gender-tailored evidence-based smoking cessation services and primary smoking prevention actions should be a priority for European healthcare policymakers to decrease tobacco-attributable lung cancer mortality.

\section{Acknowledgments}

We are grateful to the $\mathrm{WHO}$ and Eurostat and all of the competent authorities of the countries concerned for providing cause-of-death data. The WHO and Eurostat are responsible only for the provision of the original information. The presented material does not imply the expression of any opinion whatsoever on the part of the WHO, Eurostat, or other parties involved in original data transmission. The authors assume full responsibility for the analyses and interpretation of these data.

\section{Conflict of interests: none declared}

\section{Paweł Koczkodaj}

M. Sklodowska-Curie National Research Institute of Oncology

Cancer Epidemiology and Primary Prevention Department

ul. Wawelska 15 B

02-034, Warszawa, Poland

e-mail:pawel.koczkodaj@pib-nio.pl

Received: 14 Jan 2021

Accepted: 23 Jan 2021

\section{References}

1. Ferlay J, Ervik M, Lam F, Colombet M, Mery L, Piñeros M, et al. Global Cancer Observatory: Cancer Today. Lyon, France: International Agency for Research on Cancer. 2020. https://gco.iarc.fr/today (11.01.2021).

2. Ferlay J, Colombet M, Soerjomataram I, et al. Cancer incidence and mortality patterns in Europe: Estimates for 40 countries and 25 major cancers in 2018. Eur J Cancer. 2018; 103: 356-387, doi: 10.1016/j. ejca.2018.07.005, indexed in Pubmed: 30100160.

3. Ozlü T, Bülbül Y. Smoking and lung cancer. Tuberk Toraks. 2005; 53(2): 200-209.

4. Walser T, Cui X, Yanagawa J, et al. Smoking and lung cancer: the role of inflammation. Proc Am Thorac Soc. 2008; 5(8): 811-815, doi: 10.1513/ pats.200809-100TH, indexed in Pubmed: 19017734.

5. Sulkowska U, Mańczuk M, Łobaszewski J, et al. Lung cancer, the leading cause of cancer deaths among women in Europe. Nowotwory Journal of Oncology. 2015; 65(5): 395-403.

6. Segi M. Cancer Mortality for Selected Sites in 24 Countries (1950-57) Department of Public Health, Tohoku University of Medicine, Sendai 1960.

7. Kim HJ, Fay MP, Feuer EJ, et al. Permutation tests for joinpoint regression with applications to cancer rates. Stat Med. 2000; 19(3): 335-351, doi: 10.1002/(sici)1097-0258(20000215)19:3<335::aid-sim336>3.0.co;2-z, indexed in Pubmed: 10649300.

8. Vandenbroucke JP, von Elm E, Altman DG, et al. STROBE Initiative. Strengthening the Reporting of Observational Studies in Epidemiology (STROBE): explanation and elaboration. PLoS Med. 2007; 4(10): e297, doi: 10.1371/journal.pmed.0040297, indexed in Pubmed: 17941715.

9. Gallus S, Lugo A, Liu X, et al. TackSHS Project Investigators. Who Smokes in Europe? Data From 12 European Countries in the TackSHS Survey (2017-2018). J Epidemiol. 2021; 31(2): 145-151, doi: 10.2188/jea. JE20190344, indexed in Pubmed: 32249267.

10. GBD 2019 Risk Factors Collaborators. Global burden of 87 risk factors in 204 countries and territories, 1990-2019: a systematic analysis for the Global Burden of Disease Study 2019. Lancet. 2020; 396(10258): 1223-1249, doi: 10.1016/S0140-6736(20)30752-2, indexed in Pubmed: 33069327.

11. Sharbaugh MS, Althouse AD, Thoma FW, et al. Impact of cigarette taxes on smoking prevalence from 2001-2015: A report using the Behavioral and Risk Factor Surveillance Survey (BRFSS). PLoS One. 2018; 13(9): e0204416, doi: 10.1371/journal.pone.0204416, indexed in Pubmed: 30235354.

12. Ngo A, Fong GT, Craig LV, et al. Analysis of Gender Differences in the Impact of Taxation and Taxation Structure on Cigarette Consumption in 17 ITC Countries. Int J Environ Res Public Health. 2019; 16(7), doi: 10.3390/ijerph16071275, indexed in Pubmed: 30974749. 
13. Anderson SJ. Marketing of menthol cigarettes and consumer perceptions: a review of tobacco industry documents. Tob Control. 2011; 20 Suppl 2: ii20-ii28, doi: 10.1136/tc.2010.041939, indexed in Pubmed: 21504928.

14. Ford A, Moodie C, Purves R, et al. Adolescent girls and young adult women's perceptions of superslims cigarette packaging: a qualitative study. BMJ Open. 2016; 6(1): e010102, doi: 10.1136/bmjopen-2015-010102, indexed in Pubmed: 26747040.

15. Directive 2014/40/EU of the European Parliament and of the Council of 3 April 2014 on the approximation of the laws, regulations and admini- strative provisions of the Member States concerning the manufacture, presentation and sale of tobacco and related products and repealing Directive 2001/37/EC Text with EEA relevance. https://ec.europa.eu/health/sites/health/files/tobacco/docs/dir_201440_en.pdf (11.01.2021).

16. World Health Organization. The Global Health Observatory. Completeness of cause-of-death data. https://www.who.int/data/gho/data/ indicators/indicator-details/GHO/completeness-of-cause-of-death-data-(-) (11.01.2021) 